\title{
Correction to: Progerin-Induced Transcriptional Changes in Huntington's Disease Human Pluripotent Stem Cell-Derived Neurons
}

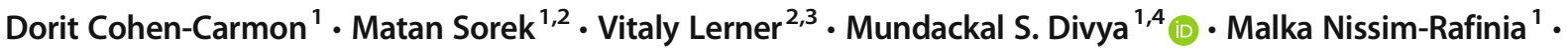 \\ Yosef Yarom $^{2,3} \cdot$ Eran Meshorer ${ }^{1,2}$ (i)
}

Published online: 15 January 2020

(C) Springer Science+Business Media, LLC, part of Springer Nature 2020

\section{Correction to: Mol Neurobiol}

https://doi.org/10.1007/s12035-019-01839-8

In the original version of the paper, the name of one of the contributing authors, Dr. Mundackal S. Divya (orcid:00000002-2869-7191), was inadvertently missing. Current affiliation of Dr. Mundackal S. Divya is Department of Pathology, Sree Chitra Tirunal Institute for Medical Sciences and Technology, Thiruvananthapuram - 695011, Kerala, India.

The correct author list is "Cohen-Carmon D, Sorek M, Lerner V, Divya MS, Nissim-Rafinia M, Yarom Y, Meshorer E”.

With the corrections above, the original article has been corrected.

Also Figure 4 legend describes Figure 3 and Figure 3 legend describes Figure 4. Therefore, The legends of Figures 3 and 4 must be switched.

The online version of the original article can be found at https://doi.org/ 10.1007/s12035-019-01839-8

Eran Meshorer

eran.meshorer@mail.huji.ac.il

1 Department of Genetics, The Alexander Silberman Institute of Life Sciences, Edmond J. Safra Campus, The Hebrew University of Jerusalem, 91904 Jerusalem, Israel

2 The Edmond and Lily Safra Center for Brain Sciences, Edmond J. Safra Campus, The Hebrew University of Jerusalem, 91904 Jerusalem, Israel

3 Department of Neurobiology, The Alexander Silberman Institute of Life Sciences, Edmond J. Safra Campus, The Hebrew University of Jerusalem, 91904 Jerusalem, Israel

4 Department of Pathology, Sree Chitra Tirunal Institute for Medical Sciences and Technology, Thiruvananthapuram, Kerala 695011, India
Fig. 3 Progerin enhances HD-associated gene expression changes in terminally differentiated neurons. a Mean expression of genes in HD cells compared to WT cells expressing WT-GFP-LMNA (top) or GFPProgerin (bottom). Dashed lines represent 2-fold difference. Red dots denote the differentially expressed genes. b Sorted values of the fold change of all expressed genes between HD and WT cells, expressing either WT-GFP-LMNA (blue) or GFP-Progerin (red). c, d Fold change of upregulated (c) and downregulated (d) genes between HD and WT cells expressing either WT-GFP-LMNA (X-axes) or GFP-Progerin (Yaxes). Red, genes which are up(/down-) regulated both with and without Progerin. Black, genes which have a different trend between HD and WT. The dashed line represents no change between HD and WT without Progerin. Note that in most cases, Progerin enhanced the already existing (although non-significant without Progerin) changes in gene expression

Fig. 4 Progerin affects nuclear morphology of human fibroblasts and post-mitotic neurons. a Left: nuclear morphology in WT-LMNA-GFP expressing fibroblasts. HP $1 \gamma$ is shown in red. Right: nuclear morphology in Progerin-GFP expressing fibroblasts. HP1 $\gamma$ is shown in red. b Nuclear morphology in Progerin-GFP expressing WT (top) and HD (bottom) iPSCderived neurons. GAD65 is shown in red. Scale bar $=10 \mu \mathrm{m}$ 